\title{
DELAY TIME IN QUATERNIONIC QUANTUM MECHANICS
}

\author{
Stefano De Leo* and Gisele Ducati ${ }^{\dagger}$ \\ - Journal of Mathematical Physics 53, 022102-8 (2012) • \\ Abstract
}

In looking for quaternionic violations of quantum mechanics, we discuss the delay time for pure quaternionic potentials. The study shows in which energy region it is possible to amplify the difference between quaternionic and complex quantum mechanics.

\section{INTRODUCTION}

Many papers have introduced the general framework of quaternionic quantum mechanics [1-10. The book published in 1995 under the title Quaternionic quantum mechanics and quantum fields by Adler [11] is, today, recognized to be indispensable to a deeper understanding of the subject. It seems, however, that a more practical side has been somewhat neglected. In other words, the investigation of quantitative and qualitative differences between complex and quaternionic quantum mechanics and the consequent experimental proposals seem to be in the early stage. It was in the hope of filling this gap that the authors have recently reviewed scattering problems in quaternionic quantum mechanics 12 14. The more elementary problems, such as square-well potentials, have been carefully re-considered with the new tendency to arrive at more practical results. The main purpose was to emphasize quaternionic violations of complex quantum mechanics which could be tested in laboratory experiments. This should make the subject more useful to and accessible over the worldwide community of scientists interested in looking for the existence of quaternionic potentials.

Contrary to what is predicted by classical mechanics, in complex quantum mechanics a particle with energy $E_{0}$ is not instantaneously reflected by a potential step of height $V_{0}$ greater than the incoming energy $E_{0}$ 15, 16. Let us briefly recall the standard introduction to delay times. The center of the incident wave packet arrives at the potential discontinuity, $x=0$, at time $t=0$. During a certain interval of time around $t=0$, the wave packet is localized in the region where the potential discontinuity is. For sufficiently large times the incident wave packet disappears and the reflected wave packet propagates towards the left at a speed $\sqrt{2 E_{0} / m}$. By using the stationary phase condition

$$
\left[\frac{\mathrm{d}}{\mathrm{d} E}\left(2 \theta_{c}-\frac{\sqrt{2 m E}}{\hbar} x_{r}-\frac{E t}{\hbar}\right)\right]_{0}=0,
$$

where $2 \theta_{c}$ is the phase of the reflection coefficient,

$$
R_{c}=\frac{\sqrt{E}-i \sqrt{V_{0}-E}}{\sqrt{E}+i \sqrt{V_{0}-E}}=\exp \left[2 i \theta_{c}\right],
$$

we can calculate the position of the center of the reflected wave packet,

$$
\sqrt{\frac{m}{2 E_{0}}} x_{r}=-t+2 \hbar\left[\frac{\mathrm{d} \theta_{c}}{\mathrm{~d} E}\right]_{0} .
$$

This clearly shows a delay time in reflection given by

$$
2 \hbar\left[\frac{\mathrm{d} \theta_{c}}{\mathrm{~d} E}\right]_{0}=\frac{\hbar}{\sqrt{E_{0}\left(V_{0}-E_{0}\right)}} .
$$

*Department of Applied Mathematics, State University of Campinas, Brazil [deleo@ime.unicamp.br]

${ }^{\dagger} \mathrm{CMCC}$, Universidade Federal do ABC, Brasil [ducati@ufabc.edu.br] 
This delay time is due to the fact that, for times close to zero, the probability of presence of the particle in the classically forbidden region is not zero. The study of the delay time in quaternionic quantum mechanics will be the subject matter of this paper.

\section{ONE-DIMENSIONAL POTENTIALS AND ONE-DIMENSIONAL MOTION}

In order to understand the essential features of quaternionic quantum mechanics, we focus our attention on quaternionic one-dimensional problems. They may be studied deriving the equation of motion from the three-dimensional quaternionic Schrödinger equation [11,

$$
\hbar \partial_{t} \Phi(\boldsymbol{r}, t)=\left[i \frac{\hbar^{2}}{2 m} \nabla^{2}-i V_{1}(\boldsymbol{r}, t)-j V_{2}(\boldsymbol{r}, t)-k V_{3}(\boldsymbol{r}, t)\right] \Phi(\boldsymbol{r}, t) .
$$

If the potential only depends on one of the three spatial coordinates, say $x$, and the motion is onedimensional, $p_{y}=p_{z}=0$, the previous equation becomes

$$
i \frac{\hbar^{2}}{2 m} \varphi^{\prime \prime}(x)=\left[i V_{1}(x)+j V_{q}(x) e^{-i \rho(x)}\right] \varphi(x)-E \varphi(x) i,
$$

where

$$
V_{q}(x)=\sqrt{V_{2}^{2}(x)+V_{3}^{2}(x)} \quad \text { and } \quad \tan \rho(x)=V_{3}(x) / V_{2}(x) .
$$

Let us now analyze the stationary states for the case of a quaternionic step potential defined by

$$
\boldsymbol{h} \cdot \boldsymbol{V}(x):=\{\mathbf{0} \text { for } x<0 \text { and } \boldsymbol{h} \cdot \boldsymbol{V} \text { for } x>0\},
$$

where $\boldsymbol{h}=(i, j, k)$. To shorten notation, it is convenient to introduce the following adimensional quantities

$$
\frac{\sqrt{2 m V_{0}}}{\hbar} x=\xi, \quad \frac{V_{q}}{V_{0}}=\nu_{q}, \quad \text { and } \quad \frac{E}{V_{0}}=\epsilon,
$$

where $V_{0}=\sqrt{V_{1}^{2}+V_{q}^{2}}$. In terms of this new adimensional quantities, the differential equation for the wave function in the free potential region $\xi<0$ becomes

$$
i \varphi_{\mathrm{I}}^{\prime \prime}(\xi)=-\epsilon \varphi_{\mathrm{I}}(\xi) i
$$

For the case of incident particles coming from the left, the free potential plane wave solution is the region $\xi<0$ is 4,13

$$
\varphi_{\mathrm{I}}(\xi)=\exp [i \sqrt{\epsilon} \xi]+R \exp [-i \sqrt{\epsilon} \xi]+j \widetilde{R} \exp [\sqrt{\epsilon} \xi],
$$

where $R$ and $\widetilde{R}$ are complex coefficients to be determined by the matching conditions. The wave function in the potential region, $\xi>0$, satisfies the following differential equation

$$
i \varphi_{\mathrm{II}}^{\prime \prime}(\xi)=\left(i \sqrt{1-\nu_{q}^{2}}+j \nu_{q} e^{-i \rho}\right) \varphi_{\mathrm{II}}(\xi)-\epsilon \varphi_{\mathrm{II}}(\xi) i
$$

whose solution is [4, 13 ,

$$
\varphi_{\mathrm{II}}(\xi)=(1+j \gamma) T \exp \left[i \alpha_{-} \xi\right]+(\beta+j) \widetilde{T} \exp \left[-\alpha_{+} \xi\right] .
$$

where

$$
\alpha_{ \pm}=\sqrt{\sqrt{\epsilon^{2}-\nu_{q}^{2}} \pm \sqrt{1-\nu_{q}^{2}}}, \quad \beta=i \frac{\nu_{q} e^{i \rho}}{\epsilon+\sqrt{\epsilon^{2}-\nu_{q}^{2}}}, \quad \gamma=-i \frac{\nu_{q} e^{-i \rho}}{\epsilon+\sqrt{\epsilon^{2}-\nu_{q}^{2}}}
$$

and $T$ and $\widetilde{T}$ are complex coefficients to be determined by the matching conditions. 
For diffusion, $E>V_{0}$, we have $\alpha_{ \pm} \in \mathbb{R}_{+}$. For the case of total reflection, $E<V_{0}$, we find

$$
\begin{aligned}
& \nu_{q}<\epsilon<1 \quad: \quad \alpha_{-}=i \sqrt{\sqrt{1-\nu_{q}^{2}}-\sqrt{\epsilon^{2}-\nu_{q}^{2}}} \in i \mathbb{R}_{+} \text {and } \alpha_{+} \in \mathbb{R}_{+}, \\
& 0<\epsilon<\nu_{q}: \alpha_{-}=i\left(1-\epsilon^{2}\right)^{1 / 4} \exp \left[-\frac{i}{2} \arctan \sqrt{\frac{\nu_{q}^{2}-\epsilon^{2}}{1-\nu_{q}^{2}}}\right] \in \mathbb{C}_{+} \text {and } \alpha_{+}=i \alpha_{-}^{*} .
\end{aligned}
$$

\section{TOTAL REFLECTION: PHASE CALCULATION}

To obtain the reflection coefficient, we have to use the matching conditions at $x=0$, i.e. $\varphi_{\mathrm{I}}(0)=\varphi_{\mathrm{II}}(0)$ and $\varphi_{\mathrm{I}}^{\prime}(0)=\varphi_{\mathrm{II}}^{\prime}(0)$. These conditions lead to the following quaternionic system,

$$
\begin{aligned}
1+R+j \widetilde{R} & =(1+j \gamma) T+(\beta+j) \widetilde{T} \\
\sqrt{\epsilon}[i(R-1)-j \widetilde{R}] & =-(1+j \gamma) T i \alpha_{-}+(\beta+j) \widetilde{T} \alpha_{+} .
\end{aligned}
$$

Multiplying the first equation for $\sqrt{\epsilon}$ and then summing it with the second equation, we find

$$
\sqrt{\epsilon}[1+R+i(R-1)]=(1+j \gamma) T\left(\sqrt{\epsilon}-i \alpha_{-}\right)+(\beta+j) \widetilde{T}\left(\sqrt{\epsilon}+\alpha_{+}\right) .
$$

The left hand of this equation is complex. Consequently, the pure quaternionic part of the right hand equation has to be zero. This immediately implies the following relation between $T$ and $\widetilde{T}$,

$$
\widetilde{T}=-\gamma \frac{\sqrt{\epsilon}-i \alpha_{-}}{\sqrt{\epsilon}+\alpha_{+}} T .
$$

By using this relation in Eqs.(11) and taking the ratio of the complex parts, after simple algebraic manipulations, we find the following reflection coefficient

$$
R=\frac{\left(\sqrt{\epsilon}+\alpha_{+}\right)\left(\sqrt{\epsilon}-\alpha_{-}\right)-\beta \gamma\left(\sqrt{\epsilon}-i \alpha_{-}\right)\left(\sqrt{\epsilon}-i \alpha_{+}\right)}{\left(\sqrt{\epsilon}+\alpha_{+}\right)\left(\sqrt{\epsilon}+\alpha_{-}\right)-\beta \gamma\left(\sqrt{\epsilon}-i \alpha_{-}\right)\left(\sqrt{\epsilon}+i \alpha_{+}\right)}
$$

We now explicitly calculate the phase for each of the two cases which characterize total reflection. Let us begin with $\nu_{q}<\epsilon<1$. In this case, we have $(\beta \gamma)^{*}=\beta \gamma, \alpha_{-}^{*}=-\alpha_{-}$and $\alpha_{+}^{*}=\alpha_{+}$. This implies

$$
R_{>}\left(\nu_{q}\right):=R\left(\nu_{q}<\epsilon<1\right)=\exp \left[2 i \theta_{>}\left(\nu_{q}\right)\right],
$$

where

$$
\theta_{>}\left(\nu_{q}\right)=\arctan \left[\frac{\beta \gamma \alpha_{+}\left(\sqrt{\epsilon}-i \alpha_{-}\right)+i \alpha_{-}\left(\sqrt{\epsilon}+\alpha_{+}\right)}{\sqrt{\epsilon}\left(\sqrt{\epsilon}+\alpha_{+}\right)-\beta \gamma \sqrt{\epsilon}\left(\sqrt{\epsilon}-i \alpha_{-}\right)}\right] .
$$

The case $0<\epsilon<\nu_{q}$ is a little bit more complicated. Let us first observe that $\beta \gamma=\exp [-2 i \omega]$ with $\tan \omega=\sqrt{\nu_{q}^{2}-\epsilon^{2}} / \epsilon$ and recall that in this case $\alpha_{+}=i \alpha_{-}^{*}$. We then find

$$
R_{<}\left(\nu_{q}\right):=R\left(0<\epsilon<\nu_{q}\right)=-\exp \left[2 i \theta_{<}\left(\nu_{q}\right)\right]
$$

where

$$
\theta_{<}\left(\nu_{q}\right)=\arctan \left(\frac{\epsilon \sin \omega+\sqrt{\epsilon} \operatorname{Im}\left[i \alpha_{-}^{*} e^{i \omega}\right]}{\left|\alpha_{-}\right|^{2} \sin \omega-\sqrt{\epsilon} \operatorname{Re}\left[\alpha_{-} e^{i \omega}\right]}\right) .
$$

The phase for a pure complex potential (standard quantum mechanics) is obtained from Eq.(13) by setting $\nu_{q}=0$,

$$
\theta_{c}:=\theta_{>}(0)=-\arctan \sqrt{\frac{1-\epsilon}{\epsilon}}
$$

For a pure quaternion potential, by using Eq.(14) for $\nu_{q}=1$, we find

$$
\theta_{q}:=\theta_{<}(1)=\arctan \left(\frac{2 \alpha \epsilon+2 \alpha^{2} \sqrt{\epsilon}+\epsilon^{3 / 2}}{4 \alpha^{3}+2 \alpha^{2} \sqrt{\epsilon}-\epsilon^{3 / 2}}\right) .
$$




\section{TOTAL REFLECTION: DELAY TIME CALCULATION}

The quaternionic wave function which determines the particle dynamics in region $\mathrm{I}, \xi<0$, is given by

$$
\varphi_{\mathrm{I}}(\xi, \tau)=(\underbrace{\exp [i \sqrt{\epsilon} \xi]}_{\varphi_{\mathrm{I}, \text { inc }}}+\underbrace{R \exp [-i \sqrt{\epsilon} \xi]+j \widetilde{R} \exp [\sqrt{\epsilon} \xi]}_{\varphi_{\mathrm{I}, \text { ref }}}) \exp [-i \epsilon \tau],
$$

where $\tau=V_{0} t / \hbar$. As observed in the introduction, for sufficiently large times the incident wave packet $\left(\varphi_{\mathrm{I}, \text { inc }}\right)$ disappears and the reflected wave packet $\left(\varphi_{\mathrm{I}, \text { ref }}\right)$ propagates towards the left. Observing that the pure quaternionic part decreases exponentially, the stationary phase method can be directly applied to the complex part of the reflected quaternionic wave function,

$$
\left[\frac{\mathrm{d}}{\mathrm{d} \epsilon}\left(2 \theta-\sqrt{\epsilon} \xi_{r}-\epsilon \tau\right)\right]_{0}=0 .
$$

The delay time is then given by

$$
\tau_{0}=2\left[\frac{\mathrm{d} \theta}{\mathrm{d} \epsilon}\right]_{0} .
$$

From this equation, by using the phase given in Eq.(15), we obtain the the standard delay time for complex potentials, i.e.

$$
\tau_{0, c}\left[\epsilon_{0, c}\right]=2\left[\frac{\mathrm{d} \theta_{c}}{\mathrm{~d} \epsilon}\right]_{0}=\frac{1}{\sqrt{\epsilon_{0, c}\left(1-\epsilon_{0, c}\right)}},
$$

where $\epsilon_{0, c}=E_{0} / V_{1}$. The dependence of $\tau_{0, c}=V_{1} t_{0} / \hbar$ upon the ratio between the incoming energy, $E_{0}$, and the complex potential, $V_{1}$, is plotted in Fig. 1 (continuous line). The minimum in the plane $\epsilon_{0, c^{-}} \tau_{0, c}$ is found at

$$
\left\{\frac{\widetilde{E}_{0, c}}{V_{1}}, \frac{V_{1} \widetilde{t}_{0, c}}{\hbar}\right\}=\{0.5,2\} .
$$

Consequently,

$$
\left\{\frac{\widetilde{E}_{0} \widetilde{t}_{0}}{\hbar}\right\}_{c}=1
$$

For a pure quaternionic potential, we have to use the phase given in Eq.(16). The expression for the quaternionic delay time is a little bit more complicated,

$$
\tau_{0, q}\left[\epsilon_{0, q}\right]=2\left[\frac{\mathrm{d} \theta_{q}}{\mathrm{~d} \epsilon}\right]_{0}=\frac{2+\frac{1}{\sqrt{2 \epsilon_{0, q}}\left(1-\epsilon_{0, q}^{2}\right)^{3 / 4}}+\frac{2 \epsilon_{0, q}}{\left(1-\epsilon_{0, q}^{2}\right)^{1 / 2}}+\frac{2 \sqrt{2 \epsilon_{0, q}}}{\left(1-\epsilon_{0, q}^{2}\right)^{1 / 4}}}{\epsilon_{0, q}+\sqrt{2 \epsilon_{0, q}}\left(1-\epsilon_{0, q}^{2}\right)^{1 / 4}+\left(1-\epsilon_{0, q}^{2}\right)^{1 / 2}},
$$

where $\epsilon_{0, q}=E_{0} / V_{q}$. The plot of $\tau_{0, q}=V_{q} t_{0} / \hbar$ as a function of the ratio between the incoming energy, $E_{0}$, and the modulus of the pure quaternionic potential, $V_{q}$, is shown in Fig. 1 (dotted line). Due to the fact that the minimum of the quaternionic delay time,

$$
\left\{\frac{\widetilde{E}_{0, q}}{V_{q}}, \frac{V_{q} \widetilde{t}_{0, q}}{\hbar}\right\}=\{0.365,2.763\},
$$

is different from the complex case, it seems simple to recognize a pure quaternionic potential by calculating the quantity $\widetilde{E}_{0} \widetilde{t}_{0} / \hbar$. Surprisingly,

$$
\left\{\frac{\widetilde{E}_{0} \widetilde{t}_{0}}{\hbar}\right\}_{q} \approx 1.0085
$$

which is very close to the result found for the complex case. Thus, an experiment involving this measurement does not represent the best choice to see quaternionic potentials. 


\section{CONCLUSIONS}

As observed in the previous section, a calculation of $\widetilde{E}_{0} \widetilde{t}_{0} / \hbar$ done by two observers which are respectively working with a complex and a pure quaternionic potential step practically gives the same result. In looking for the energy region which could amplify the difference between complex and quaternionic quantum mechanics, it is interesting to introduce new energy and time variables defined in terms of the incoming energy, $\widetilde{E}_{0}$, for which we have a minimal delay time. In terms of these new variables,

$$
\widetilde{\epsilon}_{0}=E_{0} / \widetilde{E}_{0} \text { and } \widetilde{\tau}_{0}=\widetilde{E}_{0} t_{0} / \hbar
$$

we find

$$
\begin{aligned}
& \epsilon_{0, c}=\frac{\widetilde{E}_{0}}{V_{1}} \cdot \frac{E_{0}}{\widetilde{E}_{0}}=0.5 \widetilde{\epsilon}_{0}, \\
& \widetilde{\tau}_{0, c}=\frac{\widetilde{E}_{0}}{V_{1}} \cdot \tau_{0, c}\left[0.5 \widetilde{\epsilon}_{0}\right]=0.5 \tau_{0, c}\left[0.5 \widetilde{\epsilon}_{0}\right],
\end{aligned}
$$

and

$$
\begin{aligned}
& \epsilon_{0, q}=\frac{\widetilde{E}_{0}}{V_{q}} \cdot \frac{E_{0}}{\widetilde{E}_{0}}=0.365 \widetilde{\epsilon}_{0}, \\
& \widetilde{\tau}_{0, q}=\frac{\widetilde{E}_{0}}{V_{q}} \cdot \tau_{0, q}\left[0.365 \widetilde{\epsilon}_{0}\right]=0.365 \tau_{0, q}\left[0.365 \widetilde{\epsilon}_{0}\right] .
\end{aligned}
$$

Fig. 2 clearly shows that the best choice to amplify the difference between complex and pure quater-

nionic potentials is achieved for incoming energies closed to $2 \widetilde{E}_{0}$ (this energy value represents the limit case between tunneling and diffusion for complex potentials). It is also interesting to observe that a complex potential cannot completely mimic a pure quaternionic potential. Indeed, as shown in Fig. 2 due to the different shapes of the delay time curves for complex and pure quaternionic potentials, a complex potential can perfectly mimic a pure quaternionic potential at most in two cases (see intersections between the two curves shown in Fig. 2). In Table 1, we explicitly shown the delay times for reflection as function of the incoming energy (first column) for a pure quaternionic potential of height $20 \mathrm{KeV}$ (second column). In such a table, we also find the reflection delay times for complex potentials of height $18.5 \mathrm{KeV}$ (third column), $16.5 \mathrm{KeV}$ (fourth column) and $14.5 \mathrm{KeV}$ (fifth column). These complex potentials only mimic the quaternionic potential respectively for incoming energy of 2,14 and $8 \mathrm{KeV}$. This clearly shows that the reflection delay times obtained in presence of a quaternionic potential step cannot be reproduced by a complex potential step. The energy dependence of the delay times in reflection by a step potential can be then used to determine whether the step as a quaternionic nature.

The study presented in this paper represents a preliminary analysis of quaternionic delay times. Further investigations imply a generalization from plane waves to wave packets and/or from onedimensional to three-dimensional problems.

\section{ACKNOWLEDGEMENTS}

The authors thank an anonymous referee for his interesting comments and useful suggestions. One

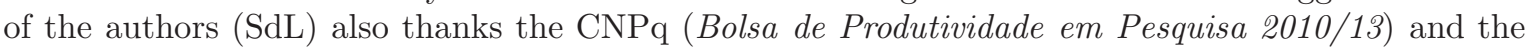
FAPESP (Grant No. 10/02213-3) for financial support.

\section{REFERENCES}

[1] A. J. Davies and B. H. McKellar, "Non-relativistic quaternionic quantum mechanics", Phys. Rev. A 40, 4209-4214 (1989).

[2] A. J. Davies and B. H. McKellar, "Observability of quaternionic quantum mechanics", Phys. Rev. A 46, 3671-3675 (1992). 
[3] S. De Leo and G. Scolarici, "Right eigenvalue equation in quaternionic quantum mechanics", J. Phys. A 33, 2971-2995 (2000).

[4] S. De Leo and G. Ducati , "Quaternionic differential operators, J. Math. Phys. 42, 2236-2265 (2001).

[5] S. De Leo, G. Scolarici and L. Solombrino, "Quaternionic eigenvalue problem", J. Math. Phys. 43, 5812-2995 (2002).

[6] S. De Leo, G. Ducati and C. Nishi, "Quaternionic potential in non-relativistic quantum mechanics, J. Phys. A 35, 5411-5426 (2002).

[7] S. De Leo and G. Ducati, "Solving simple quaternionic differential equations, J. Math. Phys. 44, 2224-2233 (2003).

[8] S. De Leo and G. Ducati, "Real linear quaternionic differential operators, Comp. Math. Appl. 48, 1893-1903 (2004).

[9] S. De Leo and G. Ducati , "Analytic plane wave solution for the quaternionic potential step", J. Math. Phys. 47, 082106-15 (2006).

[10] S. De Leo, G. Ducati, V. Leonardi, and K. Pereira, "A closed formula for the barrier transmission coefficient in quaternionic quantum mechanics", J. Math. Phys. 51, 113504-10 (2010).

[11] S. L. Adler, Quaternionic quantum mechanics and quantum fields (New York, Oxford University Press, 1995).

[12] S. De Leo and G. Ducati, "Quaternionic bound states, J. Phys. A 38, 3443-3454 (2005).

[13] S. De Leo and G. C. Ducati, "Quaternionic diffusion by a potential step, J. Math. Phys. 47, 082106-15 (2006).

[14] S. De Leo and G. C. Ducati, "Quaternionic wave packets, J. Math. Phys. 48, 052111-10 (2007).

[15] C. Cohen-Tannoudji, B. Diu and F. Lalöe, Quantum mechanics (New York, John Wiley \& Sons, 1977).

[16] E. Merzbacher, Quantum mechanics (New York, John Wiley \& Sons, 1970).

[17] E. H. Hauge and J. A. Stovneng, "Tunneling times: a critical review", Rev. Mod. Phys. 61, 917-936 (1989).

[18] V. S. Olkhovsky, E. Recami, and J. Jakiel, "Unified time analysis of photon and particle tunneling", Phys. Rep. 398, 133-178 (2004).

[19] T. E. Hartman, "Tunneling of a wave packet" , J. Appl. Phys. 33, 3427 (1962).

[20] H. Winful, "Tunneling time, the Hartman effect, and superluminality: A proposed resolution of an old paradox", Phys. Rep. 436 1-69 (2006). 


\begin{tabular}{|r|r||r|r|r|}
\hline & $V_{q}=20 \mathrm{KeV}$ & $V_{1}=18.5 \mathrm{Kev}$ & $V_{1}=16.5 \mathrm{Kev}$ & $V_{1}=14.6 \mathrm{Kev}$ \\
\hline$E_{0} / \mathrm{KeV}$ & $\mathrm{KeV} t_{0} / \hbar$ & $\mathrm{KeV} t_{0} / \hbar$ & $\mathrm{KeV} t_{0} / \hbar$ & $\mathrm{KeV} t_{0} / \hbar$ \\
\hline \hline 2 & 0.174 & $\star 0.174$ & 0.186 & 0.199 \\
\hline 4 & 0.147 & 0.131 & 0.141 & 0.154 \\
\hline 6 & 0.139 & 0.115 & 0.126 & 0.140 \\
\hline 8 & 0.138 & 0.109 & 0.121 & $\star 0.138$ \\
\hline 10 & 0.142 & 0.108 & 0.124 & 0.147 \\
\hline 12 & 0.152 & 0.113 & 0.136 & 0.179 \\
\hline 14 & 0.169 & 0.126 & $\star 0.169$ & 0.345 \\
\hline
\end{tabular}

Table 1: Reflection delay times for a pure quaternionic potential of height $V_{q}=20 \mathrm{Kev}$ (second column). The complex potentials (third, fourth and fifth column) only mimic the quaternionic potential for particular incoming energies (starred entries). Consequently, a complex potential cannot reproduce the quaternionic results. 


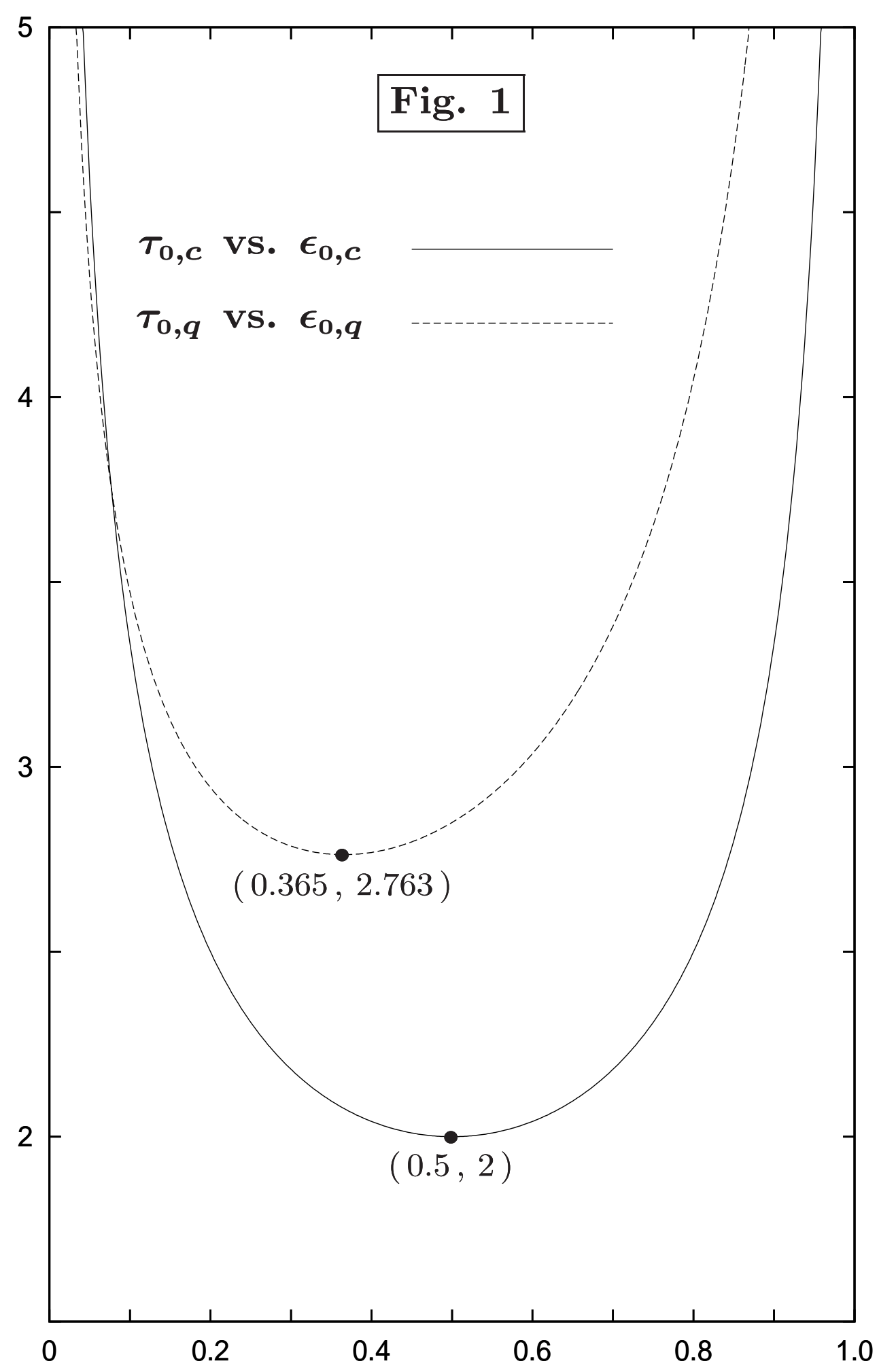

Figure 1: Delay times for complex $\left(\tau_{0, c}\right)$ and pure quaternionic $\left(\tau_{0, q}\right)$ potentials in terms of the adimensional incoming energies $E_{0} / V_{1}\left(\epsilon_{0, c}\right)$ and $E_{0} / V_{q}\left(\epsilon_{0, q}\right)$. 


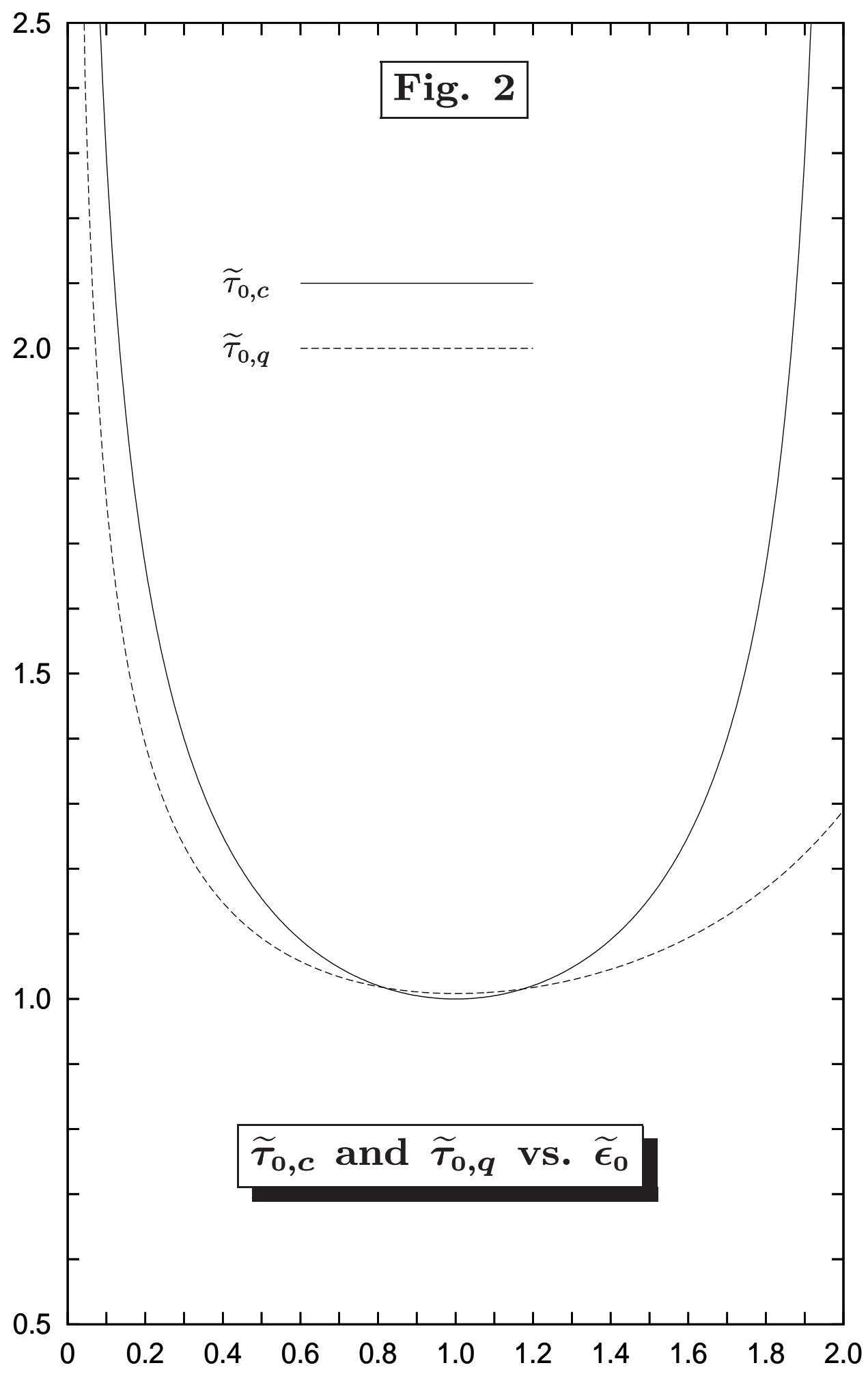

Figure 2: Delay times for complex and pure quaternionic potentials in terms of the adimensional incoming energy $E_{0} / \widetilde{E}_{0}$. The difference between quaternonic and complex quantum mechanics is amplified for incoming energies closed to $2 \widetilde{E}_{0}$. 